Bull. Austral. Math. Soc.

$34 \mathrm{~K} 15,34 \mathrm{C} 10$

VoL. 43 (1991) [147-152]

\title{
OSCILLATIONS FOR FIRST ORDER NEUTRAL DIFFERENTIAL EQUATIONS WITH VARIABLE COEFFICIENTS
}

\section{Shigui RuaN}

In this paper, sufficient conditions for oscillations of the first order neutral differential equation with variable coefficients

$$
(x(t)-c x(t-\tau))^{\prime}+p(t) x(t-\sigma)-q(t) x(t-\mu)=0
$$

are obtained, where $c, \tau, \sigma$ and $\mu$ are positive constants, $p, q \in C\left(\left[t_{0}, \infty\right), R^{+}\right)$.

\section{INTRODUCTION}

During the past decade the oscillation theory of first order neutral delay differential equations has been extensively developed by many authors. Particularly, we mention the papers by Grove, Kulennovic and Ladas [2], and Ladas and Sficas [4, 5], who investigated neutral delay differential equations with variable coefficients.

In this paper we consider the first order neutral delay differential equations with positive and negative variable coefficients

$$
(x(t)-c x(t-\tau))^{\prime}+p(t) x(t-\sigma)-q(t) x(t-\mu)=0
$$

where $t \geqslant t_{0}, c, \tau, \sigma$ and $\mu$ are positive constants, $p, q \in C\left(\left[t_{0}, \infty\right), R^{+}\right)$. Let $\left.\phi \in C\left(t_{0}-T, t_{0}\right], R\right)$, where $T=\max \{\tau, \sigma, \mu\}$. By a solution of equation (1) with initial function $\phi$ at $t_{0}$ we mean a function $x \in C\left(\left[t_{0}-T, \infty\right), R\right)$ such that $x(t)=\phi(t)$ for $t_{0}-T \leqslant t \leqslant t_{0}$. Now $x(t)-c x(t-\tau)$ is continuously differentiable for $t \geqslant t_{0}$, and $x$ satisfies equation (1) for all $t \geqslant t_{0}$. Using the method of steps, it follows that for any continuous initial function $\phi$, there exists a unique solution of equation (1) valid for $t \geqslant t_{0}$.

It is customary to define a real valued function $x(t)$ on an interval of the form $\left[t_{0}, \infty\right)$ to be oscillatory if there exists a sequence of real numbers $\left\{t_{m}\right\} \rightarrow \infty$ as $m \rightarrow \infty$ such that $t_{m} \in\left[t_{0}, \infty\right)$ and $x\left(t_{m}\right)=0, m=1,2, \ldots$ For our purpose, it is convenient to use the following definition established by Gopalsamy [1].

Received 19 March 1990

Copyright Clearance Centre, Inc. Serial-fee code: 0004-9729/91 \$A2.00+0.00. 
Definition: A real valued differentiable function $x(t)$ defined on $\left[t_{0}, \infty\right)$ is said to be oscillatory on $\left[t_{0}, \infty\right)$, if there exists a sequence $\left\{t_{m}\right\} \rightarrow \infty$ as $m \rightarrow \infty$ such that

$$
t_{m} \in\left[t_{0}, \infty\right) \text { and } x\left(t_{m}\right) x^{\prime}\left(t_{m}\right)=0, m=1,2, \ldots
$$

A real valued differentiable function $x(t)$ defined on $\left[t_{0}, \infty\right)$ is said to be nonoscillatory on $\left[t_{0}, \infty\right)$ if there exists a number $t_{1} \geqslant t_{0}$, such that

$$
x(t) x^{\prime}(t) \neq 0, \quad \forall t \geqslant t_{1} .
$$

The following lemma will be used in the proof of our results, it can be found in [3].

Lemma . Let $\rho$ be a positive constant, $h \in C\left(\left[t_{0}, \infty\right), R^{+}\right)$, and assume that

$$
\liminf _{t \rightarrow \infty} \int_{t-p}^{t} h(s) d s>\frac{1}{e}
$$

Then (a) the differential difference inequaltiy

$$
z^{\prime}(t)+h(t) z(t-\rho) \leqslant 0, \quad t \geqslant t_{0}
$$

has no eventually positive solutions;

(b) the differential difference inequality

$$
z^{\prime}(t)+h(t) z(t-\rho) \geqslant 0, \quad t \geqslant t_{0}
$$

has no eventually negative solutions.

\section{MAIN RESUltS}

THEOREM 1. Assume that

(a) $c<1, \sigma>\mu$;

(b) there exists a $t_{1} \geqslant t_{0}$, such that

$$
p(t) \geqslant q(t-(\sigma-\mu)), \quad \forall t \geqslant t_{1} \geqslant t_{0} ;
$$

(c) $\liminf _{t \rightarrow \infty} \int_{t-\sigma}^{t} p(s) d s>1 / e$;

(d) $\int_{t_{0}}^{\infty} q(s) d s<\infty$.

Then every solution of equation (1) is oscillatory.

Proof: Suppose equation (1) has a nonoscillatory solution $x(t)$, that is, there exists a number $t_{1} \geqslant t_{0}$ such that (3) holds. Without loss of generality, suppose that $x(t)>0$ for $t \geqslant t_{2} \geqslant t_{1}$. We consider the following cases: 
I. $x(t)$ is bounded on $\left[t_{2}, \infty\right)$. From (1) we have

(4) $\left[x(t)-c x(t-\tau)-\int_{t-(\sigma-\mu)}^{t} q(s) x(s-\mu) d s\right]^{\prime}+[p(t)-q(t-(\sigma-\mu))] x(t-\sigma)=0$ for all $t \geqslant t_{2}+\sigma$. Let

$$
z(t)=x(t)-c x(t-\tau)-\int_{t-(\sigma-\mu)}^{t} q(s) x(s-\mu) d s .
$$

From condition (d), $\int_{t_{0}}^{\infty} q(s) d s$ converges, and $x(t)$ is bounded, so we have

$$
\int_{t_{2}+\sigma}^{\infty} q(s) x(s-\mu) d s<\infty
$$

that is

$$
\lim _{t \rightarrow \infty} \int_{t-(\sigma-\mu)}^{t} q(s) x(s-\mu) d s=0
$$

Then, by (4), we get

$$
z^{\prime}(t)+[p(t)-q(t-(\sigma-\mu))] x(t-\sigma)=0, \quad t \geqslant t_{2}+\sigma .
$$

From condition (b) we have $z^{\prime}(t) \leqslant 0$ for all $t \geqslant t_{2}+\sigma$. Hence $z(t)$ is nonincreasing and bounded on $\left[t_{2}+\sigma, \infty\right)$, so $\lim _{t \rightarrow \infty} z(t)$ exists. Denote

$$
l=\lim _{t \rightarrow \infty} z(t)
$$

By (5), we have

$$
\lim _{t \rightarrow \infty} x(t)=\frac{l}{1-c}
$$

where $l>0$ or $l=0$.

If $l>0$, integrating (1) from $t-\sigma$ to $t$, we get

$x(t)-c x(t-\tau)-x(t-\sigma)+c x(t-\sigma-\tau)+\int_{t-\sigma}^{t} p(s) x(s-\tau) d s-\int_{t-\sigma}^{t} q(s) x(s-\mu) d s=0$.

From (7), there is a $t_{3} \geqslant t_{2}+\sigma$ such that

$$
\frac{l}{2(1-c)}<x(t)<\frac{2 l}{1-c}, \quad t \geqslant t_{3} .
$$


By (8), we have

(9)

$x(t)-c x(t-\tau)-x(t-\sigma)+c x(t-\sigma-\mu)+\frac{l}{2(1-c)} \int_{t-\sigma}^{t} p(s) d s-\frac{2 l}{1-c} \int_{t-\sigma}^{t} q(s) d s \leqslant 0$

for all $t \geqslant t_{3}+2 \sigma$. Taking the lower limit in (9) as $t \rightarrow \infty$, we get

$$
\frac{l}{2(1-c)} \int_{t-\sigma}^{t} p(s) d s \leqslant 0
$$

which contradicts condition (c).

If $l=0$, we claim that

$$
z(t)>0, \forall t \geqslant t_{2}+\sigma
$$

Indeed, since $z(t)$ is nonincreasing and bounded on $\left[t_{2}+\sigma, \infty\right)$, from conditions (c) and (d), we have

$$
\liminf _{t \rightarrow \infty} \int_{t-\sigma}^{t}[p(s)-q(t-(\sigma-\mu))] d s=\liminf _{t \rightarrow \infty} \int_{t-\sigma}^{t} p(s) d s \geqslant \frac{1}{e}
$$

so $p(t)-q(t-(\sigma-\mu)) \geqslant 0$ and is not identically and eventually zero, which means $z^{\prime}(t) \leqslant 0$ for $t \geqslant t_{2}+\sigma$ and is not identically and eventually zero. Therefore, if there is a $t_{4} \geqslant t_{2}+\sigma$ such that $z\left(t_{4}\right) \leqslant 0$, then $\lim _{t \rightarrow \infty} z(t)<0$, which contradicts $\lim _{t \rightarrow \infty} z(t)=0$. So we must have

$$
z(t)>0, t \geqslant t_{2}+\sigma
$$

On the other hand, by (6), condition (b) and the fact that $z(t) \leqslant x(t)$ for $t \geqslant t_{2}+2 \sigma$, we have

$$
z^{\prime}(t)+[p(t)-q(t-(\sigma-\mu))] z(t-\sigma) \leqslant 0, t \geqslant t_{2}+2 \sigma
$$

By the Lemma and conditions (c) and (d), (11) has no eventually positive solutions, which contradicts (10).

II. $x(t)$ is unbounded on $\left[t_{2}, \infty\right)$. From (1) we have

$$
(x(t)-c x(t-\tau))^{\prime} \leqslant q(t) x(t-\sigma), t \geqslant t_{2}+\sigma .
$$

Integrating (12) from $t_{2}+\sigma$ to $t$, we get

$$
x(t)-c x(t-\tau) \leqslant x\left(t_{2}+\sigma\right)-c x\left(t_{2}+\sigma-\tau\right)+\int_{t_{2}-\sigma}^{t} q(s) x(s-\mu) d s .
$$


Since $x(t)$ is positive and unbounded, in view of (3), we must have $x^{\prime}(t)>0$ for $t \geqslant t_{2}+\sigma$. So $x(t)$ is nondecreasing; then there exists a $t_{3} \geqslant t_{2}+\sigma$ such that

$$
x(t) \geqslant x(t-\tau), \forall t \geqslant t_{5} .
$$

Hence (13) implies that

$$
\begin{aligned}
(1-c) x(t) & \leqslant x\left(t_{2}+\sigma\right)-c x\left(t_{2}+\sigma-\tau\right)+\int_{t_{2}+\sigma-\mu}^{t-\mu} q(s+\mu) x(s) d s \\
& \leqslant x\left(t_{2}+\sigma\right)+\int_{t_{2}+\sigma-\mu}^{t} q(s+\mu) x(s) d s .
\end{aligned}
$$

that is

$$
x(t) \leqslant \frac{x\left(t_{2}+\sigma\right)}{1-c}+\int_{t_{2}+\sigma-\mu}^{t} \frac{1}{1-c} q(s+\mu) x(s) d s .
$$

By the Gronwall-Bellman inequality, we have

$$
x(t) \leqslant \frac{x\left(t_{2}+\sigma\right)}{1-c} \exp \int_{t_{2}+\sigma-\mu}^{t} \frac{1}{1-c} q(s+\mu) d s,
$$

which contradicts condition (d). The proof of Theorem 1 is now complete.

REMARK. Let $q(t)=0$ in Theorem 1; then we get Theorem 7 of Ladas and Sficas [4].

Next we consider the following equation

$$
(x(t)-c x(t-\tau))^{\prime}+\sum_{i=1}^{n} p_{i}(t) x\left(t-\sigma_{i}\right)-q(t) x(t-\mu)=0,
$$

where $c<1, \tau, \mu, \sigma_{i}(i=1,2, \ldots, n)$ are positive constants, $q, p_{i} \in C\left(\left[t_{0}, \infty\right), R^{+}\right)$, $i=1,2, \ldots, n$.

THEOREM 2. Assume that

(a) there is a $t_{1} \geqslant t_{0}, i_{0} \in\{1,2, \ldots, n\}$, such that $\sigma_{i_{0}}>\mu$, and

$$
p_{i_{0}}(t) \geqslant q\left(t-\left(\sigma_{i_{0}}-\mu\right)\right), \forall t \geqslant t_{1}
$$

(b) $\liminf _{t \rightarrow \infty} \int_{t-\sigma_{i_{0}}}^{t} p_{i_{0}}(s) d s>1 / e$;

(c) $\int_{t_{0}}^{\infty} q(s) d s<\infty$.

Then every solution of equation (14) is oscillatory.

Proof: Suppose that equation (14) has a nonoscillatory solution $x(t)$. Without loss of generality, assume that $x(t)>0$ for $t \geqslant t_{2} \geqslant t_{1} \geqslant t_{0}$; then from (14) we have

$$
(x(t)-c x(t-\tau))^{\prime}+p_{i_{0}}(t) x\left(t-\sigma_{i_{0}}\right)-q(t) x(t-\mu) \leqslant 0 .
$$

The rest of the proof is similar to that of Theorem 1 , so we omit it here. 


\section{REFERENCES}

[1] K. Gopalsamy, 'Oscillations in neutral delay-differential equations', J. Math. Phys. Sci. 21 (1987), 23-34.

[2] E.A. Grove, M.R.S. Kulennovic and G. Ladas, 'Sufficient conditions for oscillation and nonoscillation of neutral equations', J. Differential Equations 68 (1987), 373-382.

[3] R.G. Koplatadze and T.A. Canturia, 'On oscillatory and monotonic solutions of first-order differential equations with retarded arguments', Differensial'nye Uravneniya 8 (1982), 1463-1465.

[4] G. Ladas and Y.G. Sficas, 'Oscillation of neutral delay differential equations', Canad. Math. Bull. 29 (1986), 438-445.

[5] G. Ladas and Y.G. Sficas, 'Asymptotic behaviour of oscillatory solutions', Hiroshima Math. J. 18 (1988), 351-359.

[6] L. Liao, 'Oscillatory properties of a class of first order retarded differential equations', in Ordinary differential equations and control theory proceeding, Editor Z. Deng et al., pp. 237-244, 1987.

Department of Mathematics

University of Saskatchewan

Saskatoon, S7N 0W0

Canada
Present address:

Department of mathematics

University of Alberta

Edmonton, Alberta

Canada T6G 2G1 